\title{
Estratégias de enfrentamento da hospitalização em crianças avaliadas por instrumento informatizado ${ }^{1}$
}

\author{
Elissa Orlandi Moraes - Universidade Federal do Espirito Santo, Vitória, Brasil \\ Sônia Regina Fiorim Enumo - Universidade Federal do Espírito Santo, Vitória, Brasil
}

\begin{abstract}
Resumo
A hospitalização infantil pode desencadear problemas psicológicos, como depressão e estresse, sendo relevante, então, identificar as estratégias de enfrentamento utilizadas pelas crianças. Esta pesquisa analisou as estratégias de enfrentamento da hospitalização em 28 crianças hospitalizadas entre 5-20 dias, em hospital público da região Sudeste. Suas mães responderam o Child Behavior Checklist (CBCL 6-18 anos) e dados da rotina das crianças. Estas responderam, individualmente, um Instrumento de Avaliação Informatizada do Enfrentamento da Hospitalização (AEHcomp), composto por 20 cenas facilitadoras e não-facilitadoras, que permite identificar 13 estratégias de enfrentamento. No AEHcomp, ocorreram mais respostas facilitadoras à hospitalização (58,8\%). Entre 10 estratégias de enfrentamento identificadas, ruminação $(22 \%)$ e distração $(15,5 \%)$ foram mais freqüentes. Não houve correlações entre problemas de comportamento anteriores à hospitalização e comportamentos não-facilitadores; mas estes se correlacionaram inversamente à idade e diretamente às mudanças na rotina. Esta avaliação pode subsidiar intervenções preventivas de danos emocionais gerados pela hospitalização.

Palavras-chaves: Estratégias de enfrentamento; Avaliação psicológica informatizada; Hospitalização infantil.
\end{abstract}

\section{Coping to hospitalization in children assessed by IT-based instrument}

\begin{abstract}
Child hospitalization may cause psychological problems, such as depression and stress. Thus, it is important to identify the coping strategies utilized by children. This research has analyzed hospitalization coping strategies by 28 hospitalized children around 5 to 20 days in a public hospital in Brazil Southeast. Their mothers answered the Child Behavior Checklist (CBCL 6-18 years old) in addition to data from children's daily life. These children answered, individually, the Computer Assessment Instrument of Coping to Hospitalization (AEHcomp), made of 20 facilitating and non-facilitating scenes, which allow identify 13 coping strategies. AEHcomp recorded more facilitating answers to hospitalization (58.8\%). Among 10 identified coping strategies, rumination (22\%) and distraction $(15.5 \%)$ have occurred the most times. There were no correlations between behavior problems prior to the hospitalization and non-facilitating behaviors, but these were correlated inversely to age and directly to daily life changes. This evaluation can provide resources for preventive interventions in emotional damages caused by hospitalization.

Keywords: Coping; IT-based psychological assessment; Child hospitalization.
\end{abstract}

\section{Introdução}

A hospitalização de crianças relaciona-se ao surgimento de patologias associadas à própria doença, como a depressão, oriundas da limitação das funções diárias, da privação do convívio social e da anulação da individualidade, além do cumprimento indesejado de normas, regras e horários (Guimarães, 1988). Observam-se também sintomas de ansiedade e estresse em relação aos procedimentos (muitas vezes dolorosos), ao tratamento e à adaptação à doença. Tais sintomas melhoram quando os pacientes têm a oportunidade de ser acompanhados por um suporte psicológico, que proporciona uma melhor expressão e compreensão de seus sentimentos e

\footnotetext{
1 Agradecimentos à direção do Hospital Infantil de Vila Velha "Dr. Alzir Bernardino Alves" (HIABA), Vila Velha, ES às bolsistas do PIBIC/CNPq - Cibele M. C. de M. Cazelli, Kelly A. Silveira e Charlene Z. Vieira, e à Professora Doutora Alessandra Brunoro Motta. Apoio institucional: CAPES (bolsa de Mestrado); CNPq (auxílio à pesquisa Proc. n 401012/2006-9; bolsas de PIBIC).
}

conseqüentemente um melhor controle da dor, da ansiedade e da depressão (Dias, Baptista \& Baptista, 2003).

Quanto menor a criança, maior o risco de desenvolver esses sintomas em virtude da maior dificuldade de compreender a situação, pois tendem a acreditar que a doença costuma ser uma punição à desobediência (Claar, Walker \& Smith, 2002; Munhoz \& Ortiz, 2006). As internações de crianças superiores a cinco dias podem desenvolver problemas psicológicos ou de comportamento temporários, levando a alterações no desenvolvimento intelectual, afetivo e da personalidade (Dias e cols., 2003). Nessa situação, a rotina da criança pode sofrer mudanças marcadas por ambientes diferentes e no sistema biopsicossocial, implicando adaptação, dada a imposição do repouso, a limitação das atividades e a descontinuidade de suas experiências sociais, além da criança ter que aprender a lidar com novos sentimentos que surgem decorrentes da série de perdas e restrições, que podem afetar seu desenvolvimento (Munhoz \& Ortiz, 2006). Nesse 
contexto, o estudo das estratégias de enfrentamento (coping) utilizadas pelas crianças se faz importante, para ajudá-las a lidar melhor com essa situação, que impõe ansiedade e estresse.

O coping pode ser definido como um conjunto de esforços - comportamentais e cognitivos utilizado com o objetivo de lidar com situações específicas, que surgem em situações de estresse e sobrecarregam os recursos pessoais (Lazarus \& Folkman, citados por Antoniazzi, Dell'Aglio \& Bandeira, 1998). O modelo de coping adotado nesta pesquisa relaciona-se à proposição de Folkman e Lazarus (1980), que consideram quatro conceitos principais: (a) é um processo entre indivíduo e ambiente; (b) sua funcionalidade é administrar ao invés de controlar a situação aversiva; (c) seus processos implicam como a situação é percebida e interpretada pelo indivíduo; (d) compõe-se de uma mobilização de esforços cognitivos e comportamentais para administrar (reduzir, minimizar ou tolerar) os sentimentos que surgem a partir da interação com o ambiente.

As estratégias de enfrentamento investigadas nesta pesquisa refletem ações, comportamento e/ou pensamentos usados para lidar com o agente estressor, proposta esta também coerente com Compas, Connor-Smith, Saltzman, Thomsen \& Wadsworth (2001), que consideram três dimensões do coping: (a) envolve esforços ativos para alcançar algum controle pessoal sobre os aspectos estressantes do ambiente e sobre sua emoção; (b) inclui esforços para adaptar uma situação por métodos cognitivos de reestruturação, de aceitação ou de distração por meio de pensamentos e atividades positivas; (c) contém respostas de evitação ou de desligamento do fator estressor ou de sua emoção.

Os estudos de coping com crianças investigam eventos considerados estressantes, como, por exemplo, situações familiares, como o divórcio dos pais, os resultados escolares, as consultas médicas, odontológicas, e/ou a hospitalização, e situações que são difíceis de ser modificadas pela própria criança. O nível de desenvolvimento cognitivo também é um fator que influencia a utilização de determinadas estratégias (Antoniazzi e cols., 1998; Dell'Aglio, 2003; Dell'Aglio \& Hutz, 2002a, 2002b; Motta \& Enumo, 2004a, 2004b).

Os métodos de coping das crianças são mais específicos e estão de acordo com o desenvolvimento das habilidades cognitivas, sociais e regulação da emoção. Entre as estratégias mais freqüentes, identificam-se o controle do perigo, a busca de apoio, a solução de problema e a distração, enquanto as menos freqüentes envolvem agressão, autodestruição e afastamento. É interessante destacar um aspecto do coping infantil, que é o comportamento de evitação e as estratégias focalizadas na emoção, que podem funcionar de forma adaptativa quando a criança não pode mudar a situação ou quando evoca muita emoção, sendo uma tentativa de manter o controle sobre a situação (Dell'Aglio, 2003). Estudos indicam que crianças, por volta dos 6 anos de idade, usam menos estratégias de coping focalizado na emoção que crianças maiores de 9 anos, quando há um declínio significante na proporção de respostas de evitação para situações médicas e, em contrapartida, há um aumento na direção da resolução do problema. Assim como encontrado na literatura sobre adultos, as situações que evocam o coping focalizado no problema são aquelas percebidas como mais controláveis, entretanto, a situação médica evoca mais freqüentemente o coping focalizado na emoção. Band e Weisz (1988) sugerem que o coping focalizado na emoção pode se desenvolver mais devagar, em parte pela necessidade de maturidade cognitiva. As crianças menores (antes dos 6 meses) seriam as que apresentariam menor risco de perturbações perante a hospitalização por não terem estabelecido vínculos afetivos suficientemente fortes o ponto de sofrerem com uma separação relativamente curta. As crianças mais velhas conseguiriam superar melhor a separação dos pais, por estabelecerem relações estáveis com outras pessoas e também por apresentarem capacidades cognitivas eficazes para compreender melhor a necessidade dos tratamentos e para controlar os medos e a ansiedade. Muitas crianças, especialmente aquelas com menos de 4 anos de idade, demonstram alterações comportamentais temporárias após a alta (Oliveira, Dantas \& Fonsêca, 2005).

Assim, é importante lembrar que a resposta da criança à situação de hospitalização depende das estratégias de coping e de fatores inerentes à criança, como a idade e o estágio de desenvolvimento, assim como das experiências anteriores de separação da família, além das características da própria doença e da compreensão desta, e também dos hábitos familiares (Barros, 1998; Gabarra, 2005; Oliveira e cols., 2005).

No contexto da hospitalização, as crianças utilizam diversas estratégias de enfrentamento consideradas facilitadoras ou não-facilitadoras, como procurar por informações sobre a doença ou 
fazer chantagens utilizando a condição de estar doente para obter algo. Resumidamente, tais estratégias têm por objetivo manter o bem-estar do indivíduo através de mecanismos que amenizem os efeitos estressantes da doença e da hospitalização (Costa Jr., 2005; Manne, Bakeman, Jacobsen \& Redd, 1993; Motta, Enumo \& Ferrão, 2006; Peterson, 1989).

A identificação das estratégias de enfrentamento da hospitalização foi realizada a partir de próprio relato da criança, por meio de um instrumento informatizado, considerando que o computador proporciona algumas vantagens: apresentação do teste com maior clareza e qualidade, interação mais produtiva, criativa e eficiente, facilitação da comunicação e o acesso às informações produzidas pela capacidade de sintetizá-las e interpretá-las, podendo contribuir para reduzir, agilizar o trabalho de coleta dos dados, organizar o processo de aplicação do instrumento e de avaliação, permitir a manutenção de uma base única das informações, além de ser atrativo e motivar a participação das crianças (Joly, Martins, Abreu, Souza \& Cozza, 2004; Pasquali, 2001; Soprani, Leal, Enumo \& Menezes, 2005).

Assim, diante da escassez de pesquisas sobre enfrentamento em crianças no país e da relevância científica e social de se conhecer as estratégias utilizadas por crianças na hospitalização, foi proposta esta pesquisa voltada para identificar, descrever a analisar as estratégias de enfrentamento da hospitalização, por meio de instrumento informatizado, procurando, mais especificamente, identificar os comportamentos facilitadores e não-facilitadores do enfrentamento da hospitalização e suas relações com as estratégias de enfrentamento, com as alterações na rotina da criança e seus problemas de comportamento prévios à hospitalização.

\section{Método}

\section{Participantes e local de coleta de dados}

Participaram desta pesquisa 28 crianças $(20$ meninos e 8 meninas), com idade entre 6 e 12 anos (média: 9 anos e 5 meses), internadas em hospital público infantil; os responsáveis pelas crianças, como informantes, assim como a gerente socioterapêutica do hospital.

As crianças foram selecionadas segundo os critérios: estar hospitalizada por cinco dias consecutivos ou mais; ter entre 6 e 12 anos de idade, apresentar qualquer doença, exceto algum tipo de câncer; e ter autorização dos responsáveis.
A coleta de dados durou 6 meses (janeiro a junho de 2006), período em que 56 crianças preencheram os critérios de inclusão na amostra; mas somente $28(50 \%)$ delas compuseram a amostra final, em virtude de: (a) ausência dos pais no momento do contato (20), impedindo a coleta de dados; e (b) problemas de áudio nas fitas de gravação (8), impossibilitando o processamento das justificativas das crianças às respostas dadas ao instrumento.

A escolaridade das crianças variou da préescola até a $7^{a}$ série do ensino fundamental. A maioria delas $(25=89,3 \%)$ estava acompanhada pela mãe ou pelo pai e residia na região metropolitana $(78,5 \%)$.

\section{Instrumentos e materiais}

Foram utilizados, na coleta de dados, os seguintes instrumentos:

1) Ficha de Identificação da Criança - com dados do prontuário do posto de enfermagem do hospital, contendo informações sobre o histórico da doença e do tratamento (tempo de internação, patologia, número de internações, existência de complicações), e sobre a vida familiar da criança (configuração familiar, quem cuida da criança em casa, e quem está com os outros filhos durante a internação da criança);

2) Protocolo de Caracterização da Rotina da Criança (PCRC) - especialmente elaborado para a pesquisa, contém 42 questões abertas e fechadas a serem respondidas pelos responsáveis pela criança, com o objetivo conhecer a rotina antes da hospitalização e saber o que mudou durante a mesma;

3) Lista de Verificação Comportamental para Criança (Child Behavior Checklist - CBCL - 6-18 anos) (Achenbach \& Rescorla, 2004; Rocha \& Silvares, 2006) - escala composta por 138 itens: 20 relacionados à avaliação da competência social (atividades, sociabilidade e escolaridade) e 118 relacionados à avaliação de problemas de comportamento (ansiedade/depressão, isolamento/depressão, queixas somáticas, problemas sociais, problemas de pensamento, problemas de atenção, comportamento transgressor e comportamento agressivo). Esses itens fornecem dados classificados em 3 escalas: de Competência Social, de Síndromes e Orientada pelo DSM-IV (classifica os dados segundo os perfis do DSM-IV). A Escala de Síndromes classifica a criança em outras duas: a) Escala de Problemas Internalizantes (caracterizada por comportamentos relativos a aspectos particulares e 
próprios da criança, incluindo as síndromes ansiedade/depressão, isolamento/depressão e queixas somáticas); e (b) Escala de Problemas Externalizantes (caracterizada pelos comportamentos dirigidos ao ambiente, incluindo comportamentos transgressores e agressivos). Esta escala fornece um índice de distúrbio total em crianças e adolescentes de 6 a 18 anos;

4) Instrumento Informatizado para Avaliação do Enfrentamento da Hospitalização - AEHcomp (Motta, 2007; Soprani \& cols., 2005) - contém: um roteiro de entrevista, com 2 perguntas abertas sobre o que a criança faz, pensa e sente no hospital e sobre o que ela gostaria de fazer durante o período que passa no hospital; e um software com 20 cenas, desenhadas e coloridas, sobre temas que retratam possíveis estratégias de enfrentamento da hospitalização, para identificar o que as crianças relatam e sentem sobre sua condição de hospitalização, permitindo avaliar suas estratégias de enfrentamento. As cenas são classificadas em duas categorias de respostas: a) 10 comportamentos facilitadores da hospitalização (por exemplo, conversar e tomar remédio); e b) 10 comportamentos não-facilitadores (por exemplo, pensar em fugir e sentir culpa). Há uma versão para meninos e outra para meninas por ser mais motivador para a criança que ela se identifique com a personagem do instrumento, apesar da aplicação impressa não ter mostrado diferenças significativas de respostas entre os sexos (Motta, 2001). Esse software é composto por um módulo de autoria, que permite a criação de testes psicológicos para execução e coleta de dados; e um módulo avaliador, que possibilita a categorização dos resultados, o suporte a estudos estatísticos e consultas à base gerada a partir dos dados obtidos com a aplicação e outras informações inseridas. $\mathrm{Na}$ versão original, impressa, o AEH foi aplicado em 28 crianças entre 6 e 12 anos de idade, no ano 2000. Mostrou-se adequado e permitiu identificar a presença de comportamentos mais facilitadores (brincar, conversar, buscar informações) do que não-facilitadores (chorar, ficar triste, sentir medo) (Motta, Enumo \& Ferrão, 2006). Sua aplicação impressa dura em torno de 50 minutos (Motta, 2001). Fornece dados para intervenções psicológicas que possibilitem à criança expressar suas emoções e mudar seus comportamentos ante a hospitalização, ao tratamento e a doença.

Foram utilizados também os seguintes materiais e equipamentos: gravador de áudio, fitas cassete, filmadora manual, fitas de vídeo, notebook Athlon XP-M 2,8, 1024 de RAM, HD de 40 Gb, onde foram instaladas as ferramentas para implementação e suporte do software: Apache, MySql e PHP. Foi criado o banco de dados e instalado o ACAAPSI, papel e caneta, para anotações das informações relevantes para o estudo.

\section{Procedimento}

Foram executadas três etapas, descritas a seguir.

Etapa 1 - Identificação e caracterização das crianças a partir da visita às enfermarias do hospital, que incluiu o contato com a criança e seu responsável para verificar o interesse em participar $\mathrm{da}$ pesquisa, levantamento documental dos prontuários médicos e dos dados sociais das crianças e perguntas diretas feitas ao acompanhante da mesma.

Etapa 2 - Assinatura do termo de consentimento pelas mães e/ou responsáveis para a participação da criança na pesquisa, entrevistas com os mesmos sobre a criança e sobre a rotina desta, além da aplicação do instrumento para avaliação de problemas de comportamento da criança (CBCL).

Etapa 3 - Aplicação individualizada e gravada em áudio do AEHcomp, na Enfermaria e na Brinquedoteca do hospital.

\section{Processamento e análise de dados}

Os dados do CBCL (6-18 anos) foram processados no software Assessment Data Manager 6.5 (ADM), específico para os questionários do ASEBA (Achenbach System of Empiracally Based Assessment). A pontuação bruta de cada item é convertida em escores $\mathrm{T}$, e os resultados são organizados em escalas, com seus escores divididos em clínico, borderline e normal. As crianças referidas como borderline foram incluídas no grupo de crianças "clínicas", conforme recomendação dos autores.

Somente a última parte do Protocolo de Caracterização da Rotina da Criança (PCRC), em que a mãe e/ou responsável avalia o quanto a hospitalização afetou a rotina da criança foi processada, classificando-se as respostas como: a) "mudança no social", que inclui as respostas referentes a não brincar mais, não ver amigos e/ou parentes, não freqüentar a escola e a igreja; e b) "mudança na rotina", com respostas referentes às mudanças na alimentação, no sono, nos horários, e permanecer deitado e apático.

As justificativas das respostas ao AEHcomp foram transcritas integralmente, categorizando-as 
em 13 categorias propostas por Skinner, Edge, Altman e Sherwood (2003) e adaptadas para o contexto da hospitalização por Motta (2007): solução do problema, busca por suporte, esquiva, distração, reestruturação cognitiva, ruminação, desamparo, afastamento social, regulação da emoção, busca por informação, negação, oposição e delegação. Foi feito cálculo do índice de concordância entre 3 juízes (igual ou superior a 70\%), obtendo-se concordância entre $91 \%$ e $97,5 \%$.

$\mathrm{O}$ teste de $\mathrm{t}$ foi aplicado para verificar possíveis correlações entre a média de comportamentos facilitadores e de comportamentos não-facilitadores (AEHcomp), e as classificações de problemas de comportamento indicadas pelos escores do CBCL.

Para verificar se problemas de comportamento prévios à hospitalização interferiam no padrão de enfrentamento da hospitalização, foi feito o cruzamento das classificações das subescalas do CBCL (clínico e normal) com os dados do AEHcomp (estratégias facilitadoras e estratégias não-facilitadoras) e a significância foi avaliada por meio do teste de Qui-quadrado. Quando necessário, utilizou-se a correção de continuidade de Yates. O grau de associação existente entre essas variáveis (as subescalas do CBCL com o AEH comp) foi realizado pelo coeficiente $\mathrm{V}$ de Cramer, no qual valores baixos indicam uma pequena associação, enquanto os elevados, uma grande associação (Pestana \& Gageiro, 2003).

Uma análise multivariada (regressão logística) foi feita com os dados da ficha de identificação da Criança (sexo, idade, escolaridade, patologia, tempo de internação e internações), com o PCRC (se a rotina mudou, o quanto mudou e o que mudou) e o AEHcomp (comportamentos facilitadores e não-facilitadores).

Esta pesquisa foi aprovada pelo Comitê de Ética do Programa de Pós-Graduação em Psicologia da Universidade Federal do Espírito Santo e autorizada pelo hospital estudado, conforme as normas contidas tanto na Resolução no. 196/96, do Conselho Nacional de Saúde, quanto na Resolução no. 16/2000, do Conselho Federal de Psicologia.

\section{Resultados}

As informações obtidas nas fichas de identificação permitiram, além de caracterizar as crianças, verificar que o tempo de internação, no momento da coleta dos dados variou de 5 a 20 dias (média $=9$ dias), sendo que 12 crianças $(42,8 \%)$ estavam internadas pela primeira vez, 11 $(39,3 \%)$ pela segunda vez, e $5(17,9 \%)$ pela terceira ou mais. As cirurgias (hipospadia, apendicite e implante de ureter) foram a principal causa de internação (11 crianças ou 39,3\%); 9 crianças $(32,1 \%)$ foram internadas por causa de infecções (renal, epifísiose, abcesso e celulite na face), 5 $(17,9 \%)$ por fraturas e $3(10,7 \%)$ em decorrência de outras patologias (anemia falciforme, meningite, dengue hemorrágica).

As informações obtidas com o PCRC identificaram que a maioria dos pais e/ou responsáveis - $25(89,3 \%)$ - considerou que houve uma alteração na rotina da criança. Destes 25 pais, $12(48 \%)$ disseram que mudou muito, 10 (40\%) que mudou totalmente e $3(12 \%)$ que mudou pouco. Considerando toda a amostra (28 pais), 19 $(67,8 \%)$ avaliaram a mudança social (não brincar mais, não ver amigos e/ou parentes, não freqüentar a escola e a igreja) como mais expressiva; enquanto $9(32,2 \%)$ consideraram ter havido mudança da criança na rotina, com alterações na alimentação, sono e horários, além do fato da criança permanecer deitada e apática.

No CBCL (6-18 anos), foram referidas como clinicas: 11 crianças $(39,3 \%)$ no total da Escala de Competências; nas subescalas da Escala de Síndromes, $17(60,7 \%)$ crianças no Total, sendo 16 crianças $(57,1 \%)$ para Problemas Internalizantes e 16 (57,1\%) para Problemas Externalizantes; e até 8 crianças foram referidas como clínicas em uma das cinco subescalas da Escala Orientada pelo DSMIV.

No AEHcomp, as cenas de Conversar (C9, 8 anos, menino: "Porque é legal conversar, você esfria um pouco a cabeça.") e Tomar remédio (C23, 12 anos, M: "Pra ver se fica bom e vai embora rápido.") foram escolhidas por todas as 28 crianças, sendo ambas indicativas de comportamentos facilitadores. As cenas menos escolhidas foram: Pensar em fugir (4) (C23, 12 anos, M: "Porque eu não acho certo, porque, se eu fugir, eles vão atrás de mim.”) e Pensar em esconder (1) (C26, 12 anos, M: "Porque eu tenho que melhorar, prá ir embora."), ambos são comportamentos não-facilitadores (Figura 1). 


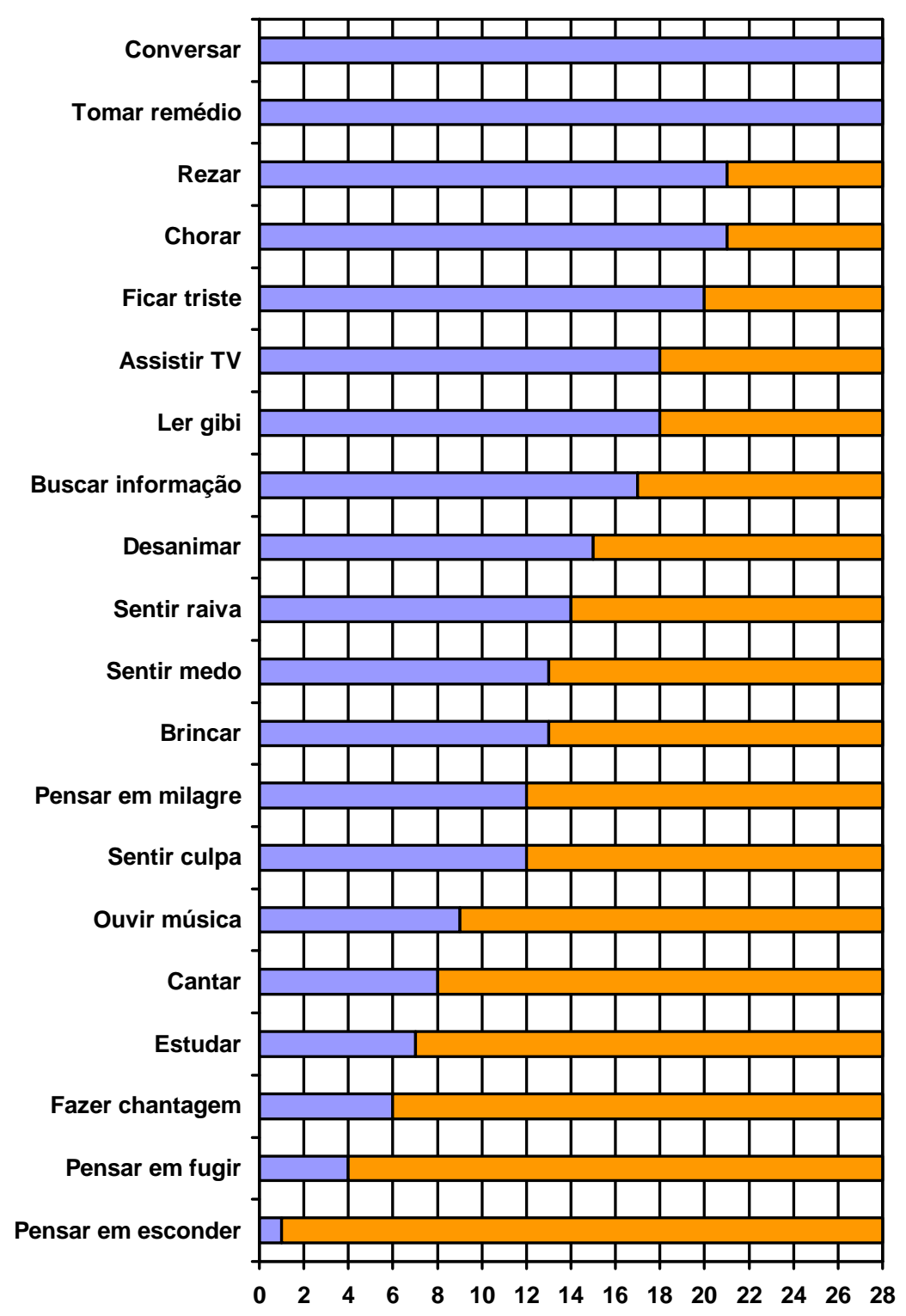

Figura 1 - Freqüência de escolha das cenas do AEHcomp por crianças hospitalizadas ( $\mathrm{n}=28)$.

As estratégias de enfrentamento da hospitalização mais freqüentes foram: Ruminação (128 justificativas) (C24, 12 anos, F, cena: Chorar: “Às vezes, sinto falta do meu pai."), seguida de Distração (90 justificativas) (C9, 8 anos, M, cena: Ler gibi: "Porque é legal, você se distrai."). As estratégias menos freqüentes foram: Afastamento social (1) e Negação (2). As estratégias de Esquiva, Desamparo e Delegação não foram escolhidas pelas crianças (Figura 2).

Possíveis problemas de comportamento prévios não interferiram no padrão de enfrentamento da hospitalização, pois não houve associação entre comportamentos facilitadores e não-facilitadores (no AEHcomp) e o T score (com classificação em clínico e não-clínico) em todas as escalas do CBCL, pelo teste do Qui-quadrado (com correção de continuidade, quando necessária).

Para analisar as relações entre os dados da ficha de identificação da criança (sexo, idade, escolaridade, tempo de internação e freqüência de internações), os dados do Protocolo de Caracterização da Rotina da Criança (se a rotina alterou, quanto alterou e o que alterou) e os dados do AEHcomp (respostas facilitadoras e nãofacilitadoras), foi feita uma regressão logística entre essas variáveis, encontrando-se relações significativas, ao nível de $5 \%$, entre as variáveis idade e o que mudou na rotina. O coeficiente 
negativo $(-0,694)$ para Idade sugere que uma criança mais nova apresentou menos comportamentos facilitadores do enfrentamento da hospitalização do que uma mais velha. O coeficiente negativo $(-3,758)$ para $O$ que mudou na rotina sugere que, quanto mais mudanças ocorrerem na rotina, provavelmente ocorrerão menos comportamentos facilitadores $\mathrm{da}$ hospitalização. O tempo de internação, o número de vezes que a criança ficou internada e o tipo de patologia que motivou a internação, bem como as outras variáveis avaliadas - sexo, o quanto a rotina mudou e escolaridade -, não mostraram significância e, portanto, não entraram no modelo estatístico. O poder explicativo do modelo é dado pelo indicador Nagelkerke $\mathrm{R}^{2} ; 52,9 \%$ das variações registradas na variável dependente são explicadas pelo conjunto de variáveis independentes.

O tempo de resposta das crianças nos instrumentos informatizados diminuiu em relação à aplicação da versão impressa do AEH, feita por Motta (2001) (de 50 minutos para 30 minutos).

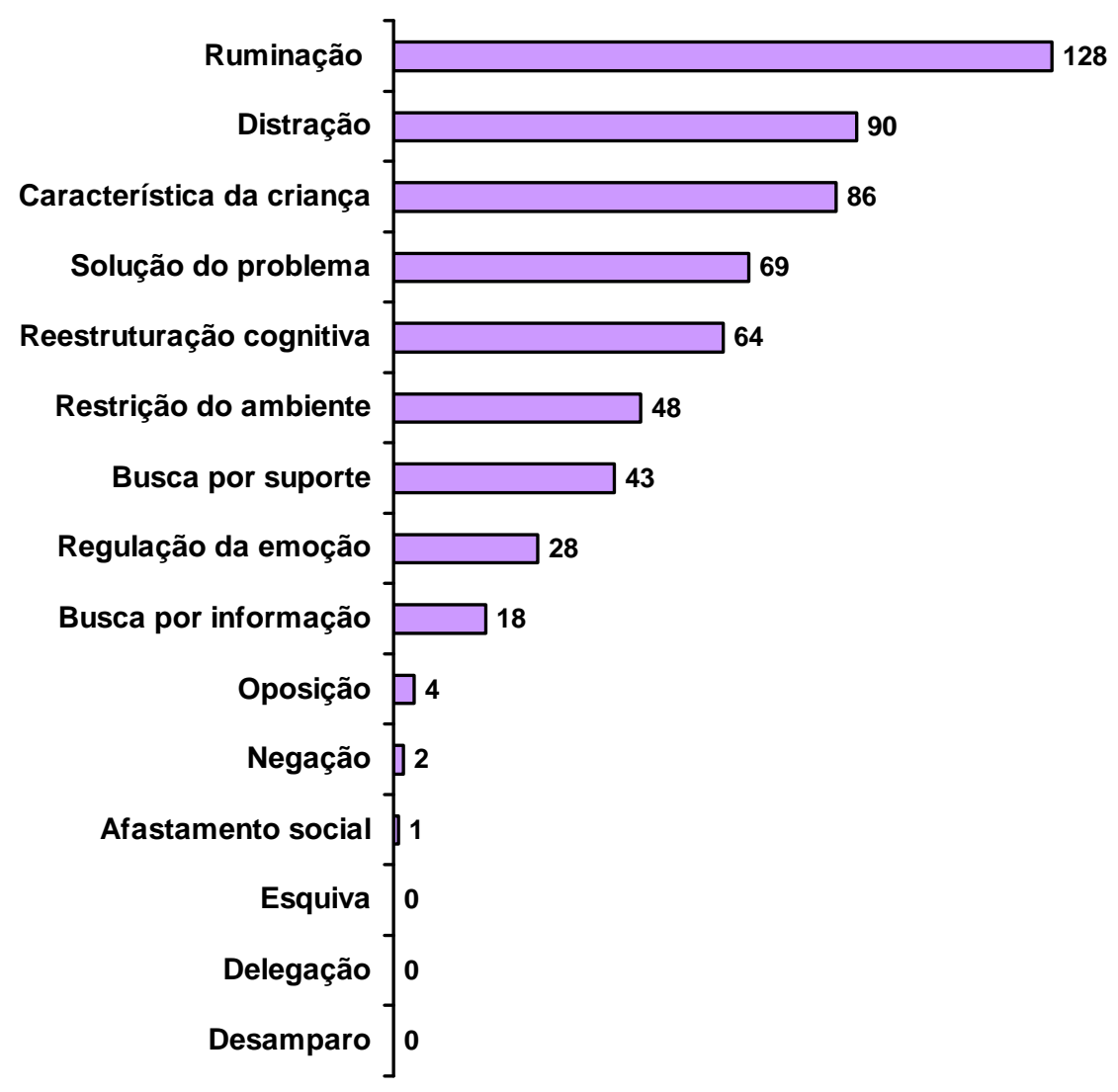

Figura 2 - Freqüência dos tipos de estratégias de enfrentamento apresentadas por crianças hospitalizadas.

\section{Discussão}

É importante lembrar que a situação estressora estudada foi a hospitalização, a qual condiciona a criança a lidar com diversas situações que podem afetar seu desenvolvimento emocional e social (Munhoz \& Ortiz, 2006). Assim, ao estudar as estratégias de enfrentamento, foi possível conhecer, pelo relato da criança, o que traz sofrimento no contexto hospitalar. Esses dados podem subsidiar os profissionais a auxiliar a criança hospitalizada a lidar melhor com essa nova situação, de forma a controlar seus níveis de ansiedade e de estresse.
O AEHcomp permitiu identificar alguns comportamentos relatados pelas crianças como relacionados ao enfrentamento das situações geradoras de estresse no hospital, com destaque para: tomar remédio, conversar, rezar, chorar e ficar triste. Os três primeiro comportamentos indicam que as crianças tendem a comportar-se de forma a fazer aquilo que lhes é permitido, sem o uso de materiais disponíveis. O comportamento de tomar remédio indica o desejo das crianças de sair desse ambiente o mais rápido possível, sendo o uso correto e obediente da medicação o meio mais rápido de conseguir a melhora. Os comportamentos de conversar e rezar sugerem a necessidade que a criança tem de buscar o apoio 
necessário para suportar as adversidades impostas pela hospitalização. Já os comportamentos de chorar e ficar triste são indicativos de que as crianças não se sentem bem durante esse período, seja pela ausência de pessoas que lhes são próximas, seja pela situação imposta, em razão da necessidade de ficar a maior parte do tempo deitada (quando não o tempo todo).

Em relação aos tipos de estratégias de enfrentamento, os resultados desta pesquisa apóiam parcialmente os dados da área (Dell'Aglio, 2003) quanto a apoio social, planejamento de soluções para problemas e distração serem as estratégias de enfrentamento mais utilizadas por crianças, pois as estratégias mais identificadas foram a ruminação e a distração. A ruminação compreende relatos indicativos de perdas impostas pela hospitalização, como a ausência de familiares, de amigos e suspensão das atividades que estava habituado a fazer. Isso pode gerar alguns transtornos associadas, como a depressão e a ansiedade em relação aos procedimentos, ou expor a criança ao risco de transtornos psicológicos, sobretudo quando a internação ultrapassar um período de cinco dias (Guimarães, 1988; Dias e cols., 2003). Esse dado revela um aspecto interessante da situação hospitalar, no qual a criança é exposta a muitas perdas (Munhoz \& Ortiz, 2006), sendo a distração uma forma de não pensar tanto no problema. A distração, como estratégia de enfrentamento, compreende atividades que as crianças relatam como prazerosas e de alivío de estresse; entre elas estão as possibilidades do brincar no hospital. O brincar é um forte aliado para o restabelecimento mais rápido da saúde da criança hospitalizada, na medida em que a criança pode manifestar sua angústia e seus medos, além de permitir que ela seja mais colaborativa nos procedimentos realizados (Motta \& Enumo, 2004a). Assim, quando a criança está mais próxima de sua situação antes da hospitalização, os problemas gerados por esta diminuem e a resposta da criança ao tratamento é melhor, sendo sua recuperação mais rápida (Munhoz \& Ortiz, 2006).

Além do sofrimento emocional, a hospitalização impõe também o sofrimento causado por mudanças no convívio social que podem desencadear ou intensificar o sofrimento imposto pela mudança de hábitos, embora existam poucos estudos sobre essas alterações (Dias e cols., 2003; Guimarães, 1988; Munhoz \& Ortiz, 2006). Contribuindo, então, nesse aspecto, foi possível identificar, entre as várias mudanças relatadas pelos pais, alterações significativas, como o afastamento dos amigos e dos familiares e o fim das atividades recreativas com a internação.

Metodologicamente, considerou-se relevante verificar se problemas prévios à internação afetariam o padrão de respostas das crianças ao AEHcomp. Nas escalas de Competências, de Síndromes, e Orientada pelo DSM-IV do CBCL (6-18 anos) (Achenbach \& Rescorla, 2004), a maioria das crianças foi referida como normal, em todas as subescalas e no total. Entretanto, na escala de Problemas, a maioria das crianças foi referida como clínica. A Escala de Problemas é gerada pela Escala de Síndromes, e essa diferença está no fato de que muitas crianças tiveram seus escores muito próximos do limite entre o clínico e o normal, e, conseqüentemente, os escores dessa escala foram levados para níveis mais baixos. De certa forma, isso indica que essas crianças precisam de algum suporte ou atendimento que lhes ajudem a lidar com as questões geradoras de estresse. Entretanto, quando se relacionaram os dados das crianças referidas como clínicas no CBCL (6-18 anos) com os comportamentos nãofacilitadores no AEHcomp, verificou-se que, estatisticamente, não houve correlações significativas que apontassem para a influência dos comportamentos prévios no padrão comportamental das crianças.

Entre as muitas variáveis que foram analisadas com possibilidades de influenciar o comportamento das crianças hospitalizadas, apenas as variáveis idade e o que mudou na rotina foram estatisticamente significativas. Esses dados confirmam pesquisas da área mostrando que a idade está relacionada com o uso de mecanismos de coping e com o controle de medo e ansiedade (Band \& Weiz, 1998; Barros, 1998; Dell'Aglio, 2003; Gabarra, 2005; Oliveira e cols., 2005).

É interessante notar que variáveis, como a patologia que provocou a internação, o tempo que a criança estava no hospital, e alguma possível experiência anterior de hospitalização não influenciaram em uma maior freqüência de comportamentos facilitadores ou não-facilitadores durante a internação em questão.

Este estudo abre portas para um novo objetivo, que é realizar estudos comparativos em hospitais de outras regiões para aprimorar as intervenções que são realizadas com as crianças hospitalizadas e, conseqüentemente, ajudá-las a enfrentar melhor e terem menos danos emocionais e comportamentais durante a hospitalização.

O material proposto é mais uma contribuição para este vasto campo de estudo e pode ajudar as 
equipes de saúde mental que trabalham com crianças hospitalizadas, pois fornecem subsídios para trabalhar com a criança, de forma a prevenir possíveis danos emocionais e/ou comportamentais gerados pela situação da hospitalização. Além disso, também indica os tipos de atividades apreciados pela criança fazendo com que o seu mundo, que ficou fora do hospital, possa acompanhá-la, a fim de minimizar o sofrimento imposto pela hospitalização.

\section{Referências}

Achenbach, T. M., \& Rescorla, L. A. (2004). Mental health practitioners' guide for the Achenbach System of Empirically Based Assessment (ASEBA). Em Laboratório de Terapia Comportamental do Instituto de Psicologia da Universidade de São Paulo (Orgs.), Guia para profissionais da saúde mental sobre o Sistema de Avaliação Empiricamente Baseado do Achenbach (ASEBA) (Laboratório de Terapia Comportamental do Instituto de Psicologia da Universidade de São Paulo, Trad.). São Paulo: Universidade de São Paulo. Trabalho não publicado.

Antoniazzi, A. S., Dell'Aglio, D. D., \& Bandeira, D. R. (1998). O conceito de coping: uma revisão teórica. Estudos de Psicologia, 3(2), 273-294.

Band, E. B. \& Weisz, J. R. (1988). How to feel better when it feels bad: Children's perspectives on coping with everyday stress. Developmental Psychology, 24(2), 247-253.

Barros, L. (1998). As conseqüências psicológicas da hospitalização infantil: Prevenção e controle. Análise Psicológica (Lisboa), 1(16), 1128 .

Claar, R. L., Walker, L. S., \& Smith, C. A. (2002). The influence of appraisals in understanding children's experiences with medical procedures. Journal of Pediatric Psychology, 27(7), 553-563.

Compas, B. E., Connor-Smith J. K., Saltzman, H., Thomsen, A. H. \& Wadsworth, M. E. (2001). Coping with stress during childhood and adolescence: Problems, progress and potencial in theory and research. Psychological Bulletin, 127(1), 87-127.

Costa Jr., A. L. (2005). Psicologia da saúde e desenvolvimento humano: $\mathrm{O}$ estudo do enfrentamento em crianças com câncer e expostas a procedimentos médicos invasivos. Em M. A. Dessen \& A. L. Costa Jr. (Orgs.). As ciências do desenvolvimento bumano: Tendências atuais e perspectivas futuras (pp 172-189). Porto Alegre: Artmed.

Dell'Aglio, D. D. (2003). O processo de coping em crianças e adolescentes: Adaptação e desenvolvimento. Temas em Psicologia, 11(1), 3845.

Dell'Aglio, D. D., \& Hutz, C. S. (2002a). Estratégias de coping e estilo atribucional de crianças em eventos estressantes. Estudos de Psicologia (Natal), 7(1), 5-13.

Dell'Aglio, D. D., \& Hutz, C. S. (2002b). Estratégias de coping de crianças e adolescentes em eventos estressantes com pares e com adultos. Psicologia USP, 13(2), 203-225.

Dias, R. R., Baptista, M. N., \& Baptista, A. S. D. (2003). Enfermaria de pediatria: Avaliação e intervenção psicológica. Em M. N. Baptista \& R. R. Dias (Orgs.). Psicologia hospitalar: Teoria, aplicações e casos clínicos (pp. 53-73). Rio de Janeiro: Guanabara Koogan.

Enumo, S. R. F. (2005). Avaliação de crianças com necessidades educativas especiais em situação de pesquisa-intervenção: Dificuldades e algumas soluções. Em H. Guilhardi \& N. C. Aguirre (Orgs.). Sobre comportamento e cognição: Expondo a variabilidade (v. 16, pp. 310-330). Santo André: ESETec.

Folkman, S. \& Lazarus, R. S. (1980). An analysis of coping in a middle-aged community sample. Journal of Health and Social Behavior, 21, 219239.

Gabarra, L. M. (2005). Crianças hospitalizadas com doenças crônicas: A compreensão da doença. Dissertação de Mestrado. Florianópolis: Universidade Federal de Santa Catarina Programa de Pós-Graduação em Psicologia.

Guimarães, S. S. (1998). A hospitalização na infância. Psicologia: Teoria e Pesquisa, 4(2), 102112.

Joly, M. C. R. A., Martins, R. X., Abreu, M. C., Souza, P. R. R., \& Cozza, H. F. P. (2004). Análise da produção científica em avaliação psicológica informatizada. Avaliação Psicológica, 3(2), 121-129.

Manne, S. L., Bakeman, R., Jacobsen, P. B., \& Redd, W. H. (1993). Children's coping during 
invasive medical procedures. Behavior Therapy, 24, 143-158.

Motta, A. B. (2001). Brincar no bospital: Câncer infantil $e$ avaliação do enfrentamento da hospitalização. Dissertação de Mestrado. Vitória: Universidade Federal do Espírito Santo.

Motta, A. B. (2007). Brincando no hospital: Uma proposta de intervenção psicológica para crianças bospitalizadas com câncer. Tese de Doutorado. Vitória: Universidade Federal do Espírito Santo - Programa de Pós-Graduação em Psicologia.

Motta, A. B., \& Enumo, S. R. F. (2004a). Brincar no hospital: Estratégia de enfrentamento da hospitalização infantil. Psicologia em Estudo, 9(1), 19-28.

Motta, A. B., \& Enumo, S. R. F. (2004b). Câncer infantil: Uma proposta de avaliação das estratégias de enfrentamento da hospitalização. Estudos de Psicologia (Campinas), 21(3), 193-202.

Motta, A. B., Enumo, S. R. F., \& Ferrão, E. S. (2006). Avaliação das estratégias de enfrentamento da hospitalização em crianças com câncer. Em M. B. M. Linhares, M. A. Crepaldi \& G. B. Perosa (Orgs.). Temas em Psicologia Pediátrica (pp. 191-217). São Paulo: Casa do Psicólogo.

Munhoz, M. A. \& Ortiz, L. C. M. (2006). Um estudo da aprendizagem e desenvolvimento em situação de internação hospitalar. Educação, 58(1), 65-83.

Pasquali, L. (2001). Testes psicológicos: Conceitos, história, tipos e usos. Em L. Pasquali (Org.). Técnicas de Exame Psicológico: TEP Manual (vol. 1, pp. 13-56). Brasília: Casa do Psicólogo.

Oliveira, G. F., Dantas, F. D. C. \& Fonsêca, P. N. (2005). O impacto da hospitalização em crianças de 1 a 5 anos de idade [Trabalho completo]. Em Sociedade Brasileira de Psicologia Hospitalar (Org.). Anais do $V$ Congresso da Sociedade Brasileira de Psicologia Hospitalar (pp. 37-54), São Paulo: SBPH.

Peterson, L. (1989). Coping by children undergoing stressful medical procedures: Some conceptual, methodological, and therapeutic issues. Journal of Consulting and Clinical Psychology, 57(3), 380-387.

Pestana, M. H. \& Gageiro, J. N. (2003). Análise de dados para Ciências Sociais - A complementaridade do SPSS (3a. ed.). Lisboa: Silabo.

Rocha, M. M., \& Silvares, E. F. M. (2006). Algumas novas formas alternativas de atendimento psicológico em clínicas-escola: Grupos recreativos. Em E. F. M. Silvares (Org.). Atendimento psicológico em clínicas-escola (pp. 91-107). Campinas, SP: Alínea.

Skinner, E. A., Edge, K., Altman, J., \& Sherwood, H. (2003). Seaching for the structure of coping: A review and critique of category systems for classifying ways of coping. Psychological Bulletin, 129(2), 216-269.

Soprani, L. S., Leal, R. N., Enumo, S. R. F. \& Menezes, C.S. (2005). Ambientes de autoria de testes e apoios a avaliações psicológicas e psicopedagógicas [Resumo completo]. Em Sociedade Brasileira de Computação (Org.), XXV Congresso da Sociedade Brasileira de Computação e XIII Workshop sobre Informática na Escola - WIE. Anais (9 pp.). São Leopoldo, RS: UNISINOS, SBC. Disponível em: http://www.unisinos.br/congresso/sbc/?sessã $\underline{\mathrm{o}=\text { wie }}$

Recebido em abril de 2008 Reformulado em agosto de 2008 Aprovado em setembro de 2008 
Sobre as autoras:

Elissa Orlandi Moraes é psicóloga (UFES, 2005) com Pós-Graduação em Psicopedagogia (Faculdade Saberes, 2006) e mestre em Psicologia pelo Programa de Pós-Graduação em Psicologia da UFES (2007). Atualmente trabalha na Associação de Pais e Amigos dos Excepcionais de Domingos Martins, (APAEDM, Espírito Santo), e é psicóloga efetiva na Secretaria de Saúde da Prefeitura Municipal de Marechal Floriano, ES, atuando com atendimento psicoterápico ambulatorial pelo SUS.

Sônia Regina Fiorim Enumo é psicóloga (PUCCamp, 1979), com mestrado em Educação Especial (UFSCar, 1985), doutorado em Psicologia Experimental (USP, 1993) e pós-doutorado (UNICAMP, 1998). É docente de Psicologia (PUCCamp, 1981-1985; UFES, desde 1986, no Depto. de Psicologia Social e do Desenvolvimento e no Programa de Pós-Graduação em Psicologia) e pesquisadora (CNPq, desde 2001) de temas como avaliação assistida, prevenção de deficiências e Estratégias de enfrentamento de doenças. 
